\title{
Robust Predictive Speed Regulation of Converter-Driven DC Motors Via A Discrete-Time Reduced-Order GPIO
}

\author{
Jun Yang, Senior Member, IEEE, Han Wu, Liang Hu, Member, IEEE, and Shihua Li, Senior Member, IEEE
}

\begin{abstract}
Converter-driven direct current (DC) motors exhibit various advantages in industry, but impose several challenges to higher-precision speed regulation in the presence of parametric uncertainties and exogenous, timevarying load torque disturbances. In this paper, the robust predictive speed regulation problem of a generic DC-DC buck converter-driven permanent magnet DC motors is addressed by using an output feedback discrete-time model predictive control (MPC) algorithm. A new discrete-time reduced-order generalized proportional-integral observer (GPIO) is proposed to reconstruct the virtual system states as well as the lumped disturbances. The estimates of GPIO are then collected for output speed prediction. An optimized duty ratio law of the converter is obtained by solving a constrained receding horizon optimization problem, where the operational constraint on control input is explicitly taken into account. Finally, the effectiveness of the proposed new algorithm is demonstrated by various experimental testing results.
\end{abstract}

Index Terms-Buck converter, DC motor, discrete-time observer, reduced-order observer, predictive speed control, multiple disturbance torques.

\section{INTRODUCTION}

$\mathbf{T}$ HE DC motor has been serving as an indispensable fundamental component of higher-precision motion control systems and related industrial instruments or devices such as renewable energy devices and electric vehicles [1]. Currently, the most popular DC motor drive is a pulse width modulation (PWM) based mechanism, that is, a PWM signal is used to regulate the armature voltage and then the speed/position of the motor. One of the remarkable advantages of such modulation mechanism is its simplicity for practical implementation [2]. However, the nonlinear switching strategy adopted here will usually lead to mutations in the armature current and voltage of the motor [3]. This may bring barriers to smooth start and control of the motor and even cause the motor damage in several extreme occasions. A simple and feasible way to address the above issues is to connect a large flat wave reactor in the armature winding of the hardware circuit [4]. In addition to the extra cost, the large capacity of the flat wave reactor will cause sluggish speed (also known as angular velocity)

J. Yang, H. Wu and S. $\mathrm{Li}$ are with School of Automation, Southeast University, Key Laboratory of Measurement and Control of CSE, Ministry of Education, Nanjing 210096, P.R. China (e-mails: j.yang84@seu.edu.cn,220151401@seu.edu.cn and Ish@seu.edu.cn).

L. Hu is with School of Computer Science and Informatics, De Montfort University, LE1 9BH UK (e-mail: liang.hu@dmu.ac.uk). responses, which is possibly unable to meet the rapidness requirement in higher-precision applications.

In the last decade, DC-DC converter gradually became a new alternative driver to the DC motor in several occasions, which mainly included buck converter [5], [6], [10], boost converter [7], [18], [19] and buck-boost converter [8]. It should be noticed that there are two manners for converterdriven motor speed regulation including unidirectional and bidirectional ones. The bidirectional speed regulation problem is extremely interesting and promising, where some very recent pioneer works on topology design of the corresponding converter-inverter driven system have been investigated in [20], [22], [24]. The new topologies bring the attractive feature of bidirectional speed regulation but impose great challenges for control design particularly in the presence of disturbances and uncertainties due to the bilinear and milti-variable properties. As such, the most favourite control approaches would be passivity-based methods [21], [23], [25], [26] which generally require fully state information and not easy to deal with various sources of external disturbances and uncertainties. Toward that end, this paper mainly focuses on unidirectional speed regulation of converter-driven DC motor system since we aim to develop an output-feedback control approach for the motor subject to various disturbances and uncertainties.

The major purposes of using DC-DC converter as drivers of DC motor can be summarized as follows: 1) it can realize smooth start and stepless speed regulation, and 2) it can achieve smooth control with reduced sudden mutation of the armature current and voltage [3]. Despite the significance mentioned above, the higher-precision speed regulation of converter-driven DC motor is quite challenging due to two reasons given as follows. Firstly, the dynamic model of converter-driven DC motor is fourth order which is much higher than second order of the traditional one. It would be difficult to expect satisfactory control performances by lower-order controllers like PID ones. Secondly, there are a large amount of disturbances and parametric uncertainties which would have different features and act on the system via different state channels. To be specific, the multiple disturbances and parametric uncertainties here would include electrical parametric perturbations of the converter, mechanical parametric variations of the motor, external time-varying load torques, etc. A general design goal of control algorithm for converter-driven DC motors is to achieve fast response, and higher accuracy of angular velocity regulation even in the presence of various sources of disturbances and uncertain- 
ties. To this end, several advanced control approaches have been investigated to enhance the performance specifications from different aspects, i.e. hierarchical/nonlinear PID control [11]-[14], [19], passivity-based control [9], [17], [18], model predictive control [27], adaptive control approach [16], [28], robust control [6], fuzzy control [29], $H_{\infty}$ control [30], and active disturbance rejection control [6], [15] for converter driven DC motor systems.

Despite the above fruitful control strategies for converterdriven DC motor systems, few of them has taken into account the control solutions to address the multiple disturbances and uncertainties. Furthermore, the control input constraint is not explicitly considered, which would cause performance degradation in several operation occasions where the hard constraint is violated. As such, in this paper we aim to propose a new discrete-time robust MPC algorithm to address the above problems. MPC has become one of the most prestigious practical control methods in industry because it can not only achieve a satisfactory tracking performance due to receding horizon optimization, but also handle control input/state constraints [31]. Thanks to the great progress of new generation of control chips with higher-computational capacity, MPC has been successfully applied in various fast dynamic systems, such as servo motors [32], converters/rectifiers [33] [34] and industrial engines [35]. In the presence of disturbances and uncertainties, it is a crucial topic on how to remove the offset within MPC systems. Generally, there are two major categories of methods to eliminate offset in MPC: 1) to augment the state model of the system by adding extra dynamics of the disturbance [36]; and 2) to represent the state space model into an incremental form for control design [37]. The principal idea of the latter one is to introduce an integral actions into the MPC to counteract disturbances and uncertainties. The utilization of integral MPCs to remove the offset would cause sacrifice of tracking control performance and bring additional efforts on parameter tuning due to performance couplings caused by integral actions.

In this paper, we investigate the offset removal approach of MPC using the former manner. To begin with, a new discretetime reduced-order GPIO is proposed to reconstruct the virtual states as well as the lumped disturbances of the converterdriven DC motor systems. The reduced-order observer is one order lower than the existing one, which facilitates the practical implementation. The construction of the observer is skillful as it can not be obtained by directly following the design procedure of the standard Luenberger observers in existing disturbance observer designs. Necessary and sufficient conditions are provided to guarantee asymptotical stability of the observer error systems as well as guide tuning of the new observer. Following the observer design, the output speed prediction is presented online by using system models as well as states and disturbance estimates. A composite cost function representing the prediction tracking error is designed by augmenting the disturbance and states estimations. By taking into account the control input constraint, a constrained duty ratio law of the converter-driven DC motor is obtained by solving a constrained receding horizon optimization problem. An initial version of the proposed algorithm appeared in [27]. However,

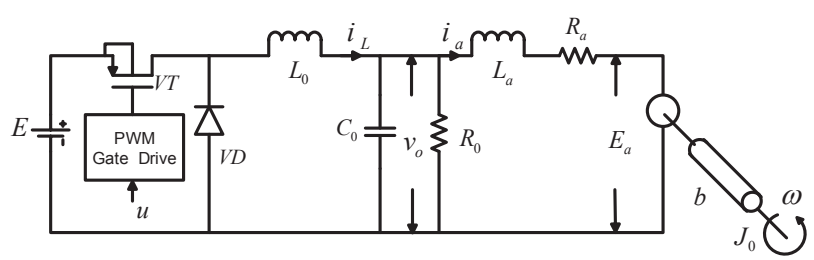

Fig. 1. The circuit diagram of DC-DC buck power converter-driven DC motor system.

the work in [27] did not include the attractive discrete-time reduced-order observer design and analysis, did not consider experimental tests as well as performance comparisons, all of which are accounted for in this paper. To conclude, the main contributions of this paper are threefold:

1) a discrete-time reduced-order GPIO is proposed for the first time with rigorous stability analysis, which has a lower order than the existing ones and facilitates practical implementation;

2) by virtue of constrained MPC design, the proposed predictive control approach obtains optimized speed regulation property even in the presence of duty ratio constraint, disturbances and uncertainties;

3) as a byproduct of the new reduced-order observer, the proposed predictive control approach saves a voltage sensor, a torque sensor as well as two current sensors as the control approach is essentially an output feedback control approach.

The proposed robust predictive speed regulation algorithm is implemented on a DSPACE-based real-time control test setup for performance validation. The experimental results illustrate that the proposed control approach exhibits superior robustness performances against various disturbances and uncertainties as compared to two popular MPC methods and the PID control approach.

\section{Dynamic Model of Buck Converter-Driven DC MOTOR SYSTEM}

\section{A. Raw Models and Control Objective}

We consider a DC-DC buck power converter acting as a smooth starter of the permanent magnet DC motor. The schematic of the converter-driven DC motor is given by Fig.1. As shown by the figure, the system consists of two parts including a generic PWM-based DC-DC buck converter and a permanent magnet DC motor. The buck converter comprises a DC input voltage source $E$, a PWM gate drive controlled switch $V T$, a diode $V D$, a filter inductor $L_{0}$, a filter capacitor $C_{0}$ and a load resistor $R_{0}$. The permanent magnet DC motor consists of an armature inductance $L_{a}$, an armature (or rotor) winding resistance $R_{a}$. The dynamic model of the overall system is given by

$$
\begin{aligned}
& L_{0} \frac{d i_{L}}{d t}=-v_{o}+u E, C_{0} \frac{d v_{o}}{d t}=i_{L}-\frac{v_{o}}{R_{0}}-i_{a}, \\
& L_{a} \frac{d i_{a}}{d t}=v_{o}-R_{a} i_{a}-k_{e} \omega, J_{0} \frac{d \omega}{d t}=k_{m} i_{a}-b \omega-\tau_{L},
\end{aligned}
$$


where $i_{L}$ is the inductor current of the buck converter, $v_{o}$ is the converter output voltage, $i_{a}$ is the armature circuit current, $\omega$ is the angular velocity of the motor shaft, $k_{e}$ is the counter electromotive force constant, $k_{m}$ is the motor torque constant, $J_{0}$ is the moment of inertia of the rotor, $b$ is the viscous friction coefficient of the motor, and $\tau_{L}$ is the load torque. The duty ratio $u \in[0,1]$ represents the control signal.

The reference angular velocity is defined as $\omega^{*}$, and the angular velocity tracking error is defined as $e=\omega-\omega^{*}$. The objective of this work is, without resorting to voltage, current and torque sensors, to design a robust predictive speed regulation algorithm such that $e \rightarrow 0$ as $t \rightarrow \infty$ in the presence of multiple sources of disturbances and uncertainties such as load torque mutations, input voltage variations, mechanical and electrical parametric perturbations.

\section{B. Model Extension and Discretization}

Defining the system states as $x=\left[x_{1}, x_{2}, x_{3}, x_{4}\right]^{T}=$ $\left[i_{L}, v_{o}, i_{a}, \omega\right]^{T}$, the dynamic models (1) can be rewritten as the following compact form

$$
\dot{x}=A x+B_{u} u+B_{d} d, y=C x,
$$

where

$$
A=\left[\begin{array}{cccc}
0 & -\frac{1}{L_{0}} & 0 & 0 \\
\frac{1}{C_{0}} & -\frac{1}{C_{0} R_{0}} & -\frac{1}{C_{0}} & 0 \\
0 & \frac{1}{L_{a}} & -\frac{R_{a}}{L_{a}} & -\frac{k_{e}}{L_{a}} \\
0 & 0 & \frac{k_{m}}{J_{0}} & -\frac{b}{J_{0}}
\end{array}\right]
$$

and $B_{u}=\left[\frac{E}{L_{0}}, 0,0,0\right]^{T}, B_{d}=\left[0,0,0,-\frac{1}{J_{0}}\right]^{T}$, and $C=$ $[0,0,0,1]$. It can be calculated that $C B_{u}=C A B_{u}=$ $C A^{2} B_{u}=0$. Taking the fourth-order time derivatives of the system output along (1) and (2), one obtains

$$
\begin{aligned}
y^{(4)}= & C x^{(4)} \\
= & C\left(A x^{(3)}+B_{u} u^{(3)}+B_{d} d^{(3)}\right) \\
= & C A\left(A x^{(2)}+B_{u} u^{(2)}+B_{d} d^{(2)}\right)+C B_{d} d^{(3)} \\
= & C A^{2}\left(A \dot{x}+B_{u} \dot{u}+B_{d} \dot{d}\right) \\
& +C A B_{d} d^{(2)}+C B_{d} d^{(3)} \\
= & C A^{3}\left(A x+B_{u} u+B_{d} d\right) \\
& +C A^{2} B_{d} \dot{d}+C A B_{d} d^{(2)}+C B_{d} d^{(3)} .
\end{aligned}
$$

Denoting $f=C A^{4} x+C A^{3} B_{d} d+C A^{2} B_{d} \dot{d}+C A B_{d} \ddot{d}+$ $C B_{d} d^{(3)}$ as the lumped disturbances and $m=C A^{3} B_{u}$ as the control coefficient, one has the following input-output formulation

$$
y^{(4)}=f+m u,
$$

Letting $z=\left[z_{1}, z_{2}, z_{3}, z_{4}\right]^{T}=[y, \dot{y}, \ddot{y}, \dddot{y}]^{T}$, one obtains the following controllable state-space model

$$
\dot{z}=A_{m} z+B_{u m} u+B_{f m} f, y=C_{m} z,
$$

where $C_{m}=\left[\begin{array}{llll}1 & 0 & 0 & 0\end{array}\right]$ and

$$
A_{m}=\left[\begin{array}{cccc}
0 & 1 & 0 & 0 \\
0 & 0 & 1 & 0 \\
0 & 0 & 0 & 1 \\
0 & 0 & 0 & 0
\end{array}\right], B_{u m}=\left[\begin{array}{c}
0 \\
0 \\
0 \\
m
\end{array}\right], B_{f m}=\left[\begin{array}{l}
0 \\
0 \\
0 \\
1
\end{array}\right] \text {. }
$$

To facilitate the controller design, the discretization of model (5) is carried out as follows. The discretization of continuoustime model is obtained in a sampled-data manner through zero-order holders, which is commonly used in the computer controlled systems [37]. To this end, the discrete-time statespace model of (5) can be obtained and given by

$$
z_{k+1}=A_{d} z_{k}+B_{u d} u_{k}+B_{f d} f_{k}, y_{k}=C_{d} z_{k},
$$

where the system matrices are given by $A_{d}=e^{A_{m} T_{S}}, B_{u d}=$ $\int_{0}^{T_{S}} e^{A_{m} \tau} d \tau \cdot B_{u m}, B_{f d}=\int_{0}^{T_{S}} e^{A_{m} \tau} d \tau \cdot B_{f m}, C_{d}=C_{m}$, and $T_{s}$ represents the sampling period.

For the purpose of jointly states and disturbance estimation, we define the lumped disturbance and its derivative as two augmented states. As such, by denoting a new state vector $\bar{z}=\left[z_{1}, z_{2}, z_{3}, z_{4}, z_{5}, z_{6}\right]^{T}=[y, \dot{y}, \ddot{y}, \dddot{y}, f, \dot{f}]^{T}$, one can get an extended state-space model below

$$
\dot{\bar{z}}=A_{e} \bar{z}+B_{e} u_{k}, y=C_{e} \bar{z},
$$

where $C_{e}=\left[\begin{array}{llllll}1 & 0 & 0 & 0 & 0 & 0\end{array}\right]$, and

$$
A_{e}=\left[\begin{array}{llllll}
0 & 1 & 0 & 0 & 0 & 0 \\
0 & 0 & 1 & 0 & 0 & 0 \\
0 & 0 & 0 & 1 & 0 & 0 \\
0 & 0 & 0 & 0 & 1 & 0 \\
0 & 0 & 0 & 0 & 0 & 1 \\
0 & 0 & 0 & 0 & 0 & 0
\end{array}\right], B_{e}=\left[\begin{array}{c}
0 \\
0 \\
0 \\
m \\
0 \\
0
\end{array}\right] .
$$

By utilizing the same discretization process as above, a discrete-time state-space model of the extended system (7) is obtained and given by

$$
\bar{z}_{k+1}=\bar{A} \bar{z}_{k}+\bar{B} u_{k}, y_{k}=\bar{C} \bar{z}_{k},
$$

where the system matrices are given by $\bar{A}=e^{A_{e} T_{S}}, \bar{B}=$ $\int_{0}^{T_{S}} e^{A_{e} \tau} d \tau \cdot B_{e}$, and $\bar{C}=C_{e}$.

\section{Controller Design}

\section{A. Discrete-Time Reduced-Order GPIO}

In this subsection, we aim to design a discrete-time reducedorder GPIO to estimate the reduced-order states as well as disturbance information. Toward that end, the extended unmeasurable states to be observed can be defined as $w=$ $\left[z_{2}, z_{3}, z_{4}, z_{5}, z_{6}\right]^{T}$, given by

$$
w_{k}=L \bar{z}_{k}
$$

where

$$
L=\left[\begin{array}{llllll}
0 & 1 & 0 & 0 & 0 & 0 \\
0 & 0 & 1 & 0 & 0 & 0 \\
0 & 0 & 0 & 1 & 0 & 0 \\
0 & 0 & 0 & 0 & 1 & 0 \\
0 & 0 & 0 & 0 & 0 & 1
\end{array}\right]
$$

A new discrete-time reduced-order observer for the system (8) based on the angular velocity $y_{k}$ and the duty ratio $u_{k}$ is designed as

$$
\left\{\begin{aligned}
\xi_{k+1} & =F \xi_{k}+G y_{k}+H u_{k}, \\
\hat{w}_{k} & =\xi_{k}+N y_{k},
\end{aligned}\right.
$$

where $\hat{w}_{k}$ is the estimate of the state $w_{k}$. 
The following lemma explains the existence condition for the observer, whose proof can be found from [38], [39] and is omitted here for space.

Lemma 1: Suppose that the matrix pair $\{F, G\}$ is controllable and the matrix pair $\{\bar{A}, \bar{C}\}$ is observable. There exists a nonsingular matrix $P$ such that the following Sylvester equation holds

$$
\left\{\begin{aligned}
P \bar{A}-F P & =G \bar{C}, \\
H & =P \bar{B} .
\end{aligned}\right.
$$

The stability of the proposed observer is presented in the following theorem.

Theorem 1: Suppose that the matrices $F, G, H$ and $N$ are designed such that the following three conditions hold:

1) the eigenvalues of $F$ lies inside the unit circle, and the pair $\{F, G\}$ is controllable;

2) the matrices $F, G$ and $H$ satisfy the Sylvester equation given by (11);

3) $N$ is designed such that

$$
L-P-N \bar{C}=0 .
$$

The estimate $\hat{w}_{k}$ generated by the observer (10) will asymptotically converge to the reduced-order extended state $w_{k}$ given in (9).

Proof: To begin with, define the error of the observer as

$$
\varepsilon_{k}=P \bar{z}_{k}-\xi_{k} .
$$

It can be deduced from (8), (10), (11) and (13) that the error dynamics of the observer are governed by

$$
\begin{aligned}
\varepsilon_{k+1}= & F \varepsilon_{k}+(P \bar{A}-F P-G \bar{C}) \bar{z}_{k} \\
& +(P \bar{B}-H) u_{k} \\
= & F \varepsilon_{k},
\end{aligned}
$$

which is asymptotically stable as the eigenvalues of $F$ have been assigned within the unit circle. In what follows, the estimation error $e_{k}=w_{k}-\hat{w}_{k}$ of the proposed observer is evaluated. Invoking (8), (9), (10), (13) and (12) gives

$$
\begin{aligned}
e_{k} & =w_{k}-\hat{w}_{k} \\
& =L \bar{z}_{k}-\left(\xi_{k}+N y_{k}\right) \\
& =L \bar{z}_{k}-\left(P \bar{z}_{k}-\varepsilon_{k}+N \bar{C} \bar{z}_{k}\right) \\
& =\varepsilon_{k}+(L-P-N \bar{C}) \bar{z}_{k} \\
& =\varepsilon_{k},
\end{aligned}
$$

which implies that $e_{k}$ will converge to zero as $\varepsilon_{k}$ converges to zero asymptotically. This completes the proof.

Based upon the above synthesis and analysis, the design procedure of the proposed discrete-time reduced-order GPIO is summarized as follows:

1) Design a controllable matrix pair $\{F, G\}$ with the eigenvalues of $F$ inside the unit circle;

2) Solve the nonsingular matrix $P$ from the Sylvester equation $P \bar{A}-F P=G \bar{C}$, and calculate matrix $H$ as $H=P \bar{B}$

3) Solve the matrix $N$ from $L-P-N \bar{C}=0$.

Remark 1: The reduced-order observer (10) is one order lower than the existing one, which facilitates the practical implementation. Furthermore, most of the existing GPIO is only available for full-order continuous-time domain design. The reduced-order GPIO design in discrete-time domain is quite different and proposes several challenges in synthesis, analysis and parameter tuning. To be specific, the construction of the observer is skillful as it can not be obtained by directly following the design procedure of standard Luenberger observers in existing GPIO [41], extended state observer (ESO) [42] and also other disturbance observer [43], [44] designs.

\section{B. Robust Predictive Speed Controller}

Following the construction of the reduced-order GPIO, the design of the proposed robust predictive speed controller is implemented in this part, which consists of the following three steps.

Step 1-Angular Velocity Prediction: According to the definition given by (9), the estimations of states and disturbances have been given and represented by $\hat{\omega}_{k}=\left[\hat{\dot{y}}_{k}, \hat{\ddot{y}}_{k}, \hat{\ddot{y}}_{k}, \hat{f}_{k}, \hat{\dot{f}}_{k}\right]^{T}$.

For angular velocity prediction, we define the prediction and control horizons as $N_{p}$ and $N_{c}$, respectively, which satisfy $N_{C} \leqslant N_{P}$. For predictive control law design, we also assume that in addition to the control horizon, the amount of control input remains unchanged, namely, $u_{k+i}=u_{k+N_{C}-1}$, $i=N_{C} \cdots N_{P-1}$. Let $\hat{z}_{k}=\left[y_{k}, \hat{\dot{y}}_{k}, \hat{\ddot{y}}_{k}, \hat{\ddot{y}}_{k}\right]^{T}$. The angular velocity can be then predicted by the following equation

$$
\hat{Y}_{k}=\Psi \hat{z}_{k}+\Phi_{u} U_{k}+\Phi_{f} \hat{f}_{k},
$$

where $\hat{Y}_{k}$ and $U_{k}$ are defined as $\hat{Y}_{k} \quad=\quad\left[\hat{y}_{k+1}, \hat{y}_{k+2}, \cdots, \hat{y}_{k+N_{P}}\right]^{T}, U_{k}=$ $\left[u_{k}, u_{k+1}, \cdots, u_{k+N_{C}-1}\right]^{T}$, and the matrix $\Phi_{u}$ is given by (17), whereas matrices $\Psi$ and $\Phi_{f}$ are given by

$$
\Psi=\left[\begin{array}{c}
C_{d} A_{d} \\
C_{d} A_{d}^{2} \\
\vdots \\
C_{d} A_{d}{ }^{N_{P}}
\end{array}\right], \Phi_{f}=\left[\begin{array}{c}
C_{d} B_{f d} \\
C_{d} B_{f d}+C_{d} A_{d} B_{f d} \\
\vdots \\
\sum_{i=0}^{N_{P}-1} C_{d} A^{i} B_{f d}
\end{array}\right] .
$$

Step 2-Definition of Cost Function: The speed reference signal can be presented along the prediction horizon as

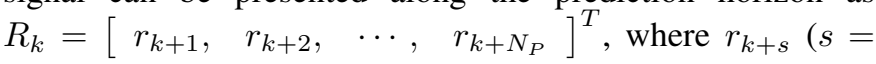
$\left.1, \cdots, N_{P}\right)$ is the reference signal of the $k$-th step of prediction. The cost function is designed by

$$
J=\left(R_{k}-\hat{Y}_{k}\right)^{T}\left(R_{k}-\hat{Y}_{k}\right) .
$$

Substituting the angular velocity prediction $\hat{Y}_{k}$ in (16) into the cost function (18), one obtains

$$
\begin{aligned}
J= & \left(R_{k}-\Psi \hat{z}_{k}-\Phi_{f} \hat{f}_{k}\right)^{T}\left(R_{k}-\Psi \hat{z}_{k}-\Phi_{f} \hat{f}_{k}\right) \\
& -2 U_{k}^{T} \Phi_{u}^{T}\left(R_{k}-\Psi \hat{z}_{k}-\Phi_{f} \hat{f}_{k}\right) \\
& +U_{k}^{T} \Phi_{u}^{T} \Phi_{u} U_{k} .
\end{aligned}
$$

Since the first term in (19) is independent on the decision variable $U_{k}$, one has

$$
\begin{aligned}
J^{*} & =\min _{U_{k}} J \\
& =\min _{U_{k}}\left(-2 U^{T} E_{P k}+U^{T}{ }_{k} H_{P} U_{k}\right),
\end{aligned}
$$




$$
\Phi_{u}=\left[\begin{array}{cccc}
C_{d} B_{u d} & 0 & \cdots & 0 \\
C_{d} A_{d} B_{u d} & C_{d} B_{u d} & \cdots & 0 \\
\vdots & \vdots & \vdots & \vdots \\
C_{d} A_{d}{ }^{N_{P}-1} B_{u d} & C_{d} A_{d}{ }^{N_{P}-2} B_{u d} & \cdots & \sum_{i=N_{C}}^{N_{P}} C_{d} A_{d}^{i-N_{c}} B_{u d}
\end{array}\right] .
$$

where $E_{P k}=\Phi_{u}{ }^{T}\left(R_{k}-\Psi \hat{z}_{k}-\Phi_{f} \hat{f}_{k}\right)$ and $H_{P}=\Phi_{u}{ }^{T} \Phi_{u}$.

Step 3-Constrained Receding Horizon Optimization: The duty ratio of the converter should be confined in the range of $u \in[0,1]$. As such, the constraints of the control input are imposed and represented as follows [40]

$$
M U_{k} \leqslant \Gamma,
$$

where

$$
M=\left[\begin{array}{cccc}
-1 & 0 & \cdots & 0 \\
1 & 0 & \cdots & 0
\end{array}\right]_{2 \times N_{C}}, \Gamma=\left[\begin{array}{l}
0 \\
1
\end{array}\right] .
$$

For practical implementation, the approach of optimization of Lagrange multipliers is utilized as it is efficent and can directly search for the active constraints. Consequently, the constrained quadratic programming $(\mathrm{QP})$ problem of (20) is equivalent to the following min-max optimization problem

$$
\max _{\lambda} \min _{U_{k}}\left[J_{y}+2 \lambda^{T}\left(M U_{k}-\Gamma\right)\right] .
$$

The cost function (22) has minimum when $U_{k}$ and $\lambda$ satisfy

$$
U_{k}^{*}=H_{p}^{-1}\left(E_{P_{k}}-M^{T} \lambda^{*}\right) .
$$

To this end, the min-max optimization problem equals to the following constrained QP problem

$$
\begin{aligned}
& \min _{\lambda}\left[\lambda^{T} M H_{p}{ }^{-1} M^{T} \lambda-2 \lambda^{T}\left(M H_{p}{ }^{-1} E_{p_{k}}-\Gamma\right)\right] \\
& \text { s.t. } \quad \lambda \geq 0 .
\end{aligned}
$$

where the terms regardless of the new decision variable $\lambda$ are neglected. The solutions of the above constrained QP problem can be denoted as

$$
\begin{gathered}
\lambda^{*}=\arg \min _{\lambda}\left[\lambda^{T} M H_{p}{ }^{-1} M^{T} \lambda-2 \lambda^{T}\left(M H_{p}{ }^{-1} E_{p_{k}}-\Gamma\right)\right] \\
\text { s.t. } \lambda \geq 0 .
\end{gathered}
$$

According to the receding horizon optimization principle, the first element of the control sequence $U_{k}$ is implemented, that is

$$
u_{k}^{*}=\left[\begin{array}{llll}
1 & 0 & \cdots & 0
\end{array}\right] U_{k}^{*} .
$$

Remark 2 (Paramter Tuning Guidelines): The tuning of prediction and control horizons should comprahensively take into account system stability, dynamic performance, robustness and computation burden [45], [46]. In general, the larger $N_{p}$ is helpful to improve stability, whereas the larger $N_{c}$ can fasten the dynamic performance but decrease robustness performance. Larger $N_{p}$ and $N_{c}$ will also increase computation burben for the control algorithm. The adjustment of the convergence rate of the observer can be manipulated by assigning the eigenvalues of matrix $F$. The eigenvalues with smaller absolute values imply faster convergence rate of the discrete-time observer. The other matrices $G, H$ and $N$ in the

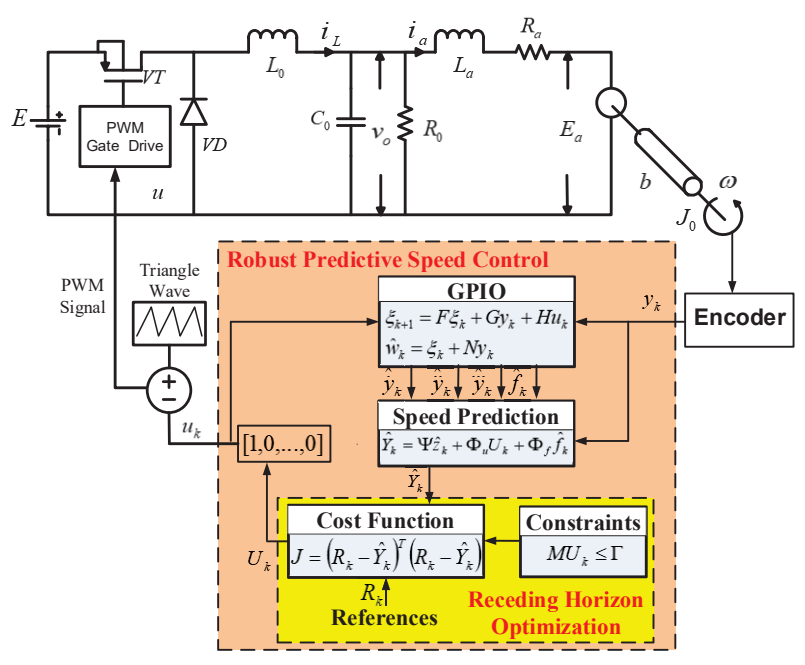

Fig. 2. The control structure of the buck converter-driven DC motor under the proposed robust predictive speed regulation algorithm.

observer are determined by the design procedures given above Remark 1.

The above constrained receding horizon optimization process is performed every sampling period to derive the optimized control law. The control structure of the proposed robust predictive speed regulation algorithm for the buck converterdriven DC motor system is shown in Fig.2.

\section{EXPERIMENTAL IMPLEMENTATION AND PERFORMANCE VALIDATION}

The configuration of the experimental test setup is shown by the top part of Fig. 3, while the real experimental prototype can be seen from the bottom part of Fig. 3. As shown by Fig. 3, the experimental test setup consists of a permanent magnet DC motor, a DC-DC buck power converter, a magnetic powder brake torque adjusting device, a tension controller, a DC power supply device, an encoder, and a DS1103 DSPACE single board system, etc. The main part of the controller is built by modules in MATLAB/Simulink library. The proposed discrete-time robust predictive speed controller is implemented by using the MATLAB Function template for programming. The sampling frequency as well as the controller updating frequencies of the experiment is setting as $f_{s}=3.3 \mathrm{kHz}$. The fixed PWM switching frequency of the converter is designed as $f_{\text {pwm }}=10 \mathrm{kHz}$. The nominal values of the parameters of converter-driven DC motor system are listed in Table I.

To validate the feasibility and efficacy of the proposed robust predictive speed regulation approach, we have conducted thorough experimental comparisons with the PID controller 


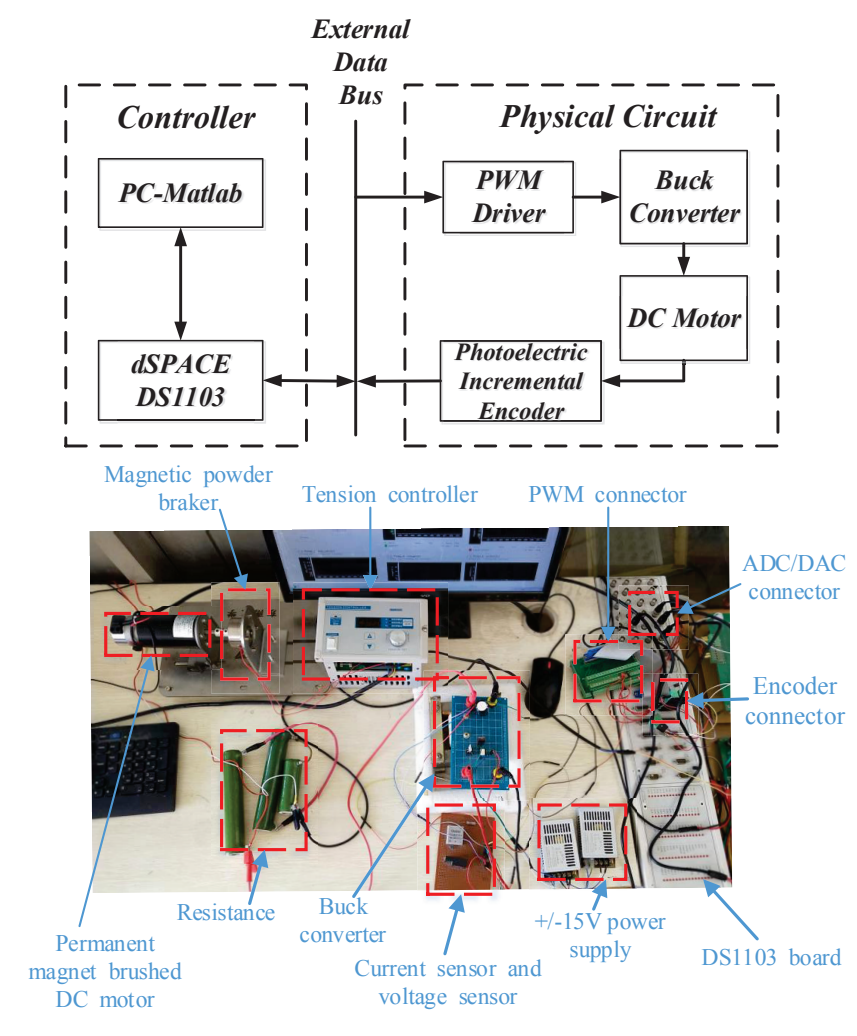

Fig. 3. Configuration of experimental setup (top), and photograph of the experimental prototype (bottom).

TABLE I

Parameters of The ConVERTER-Driven DC Motor

\begin{tabular}{l|c|c}
\hline Parameters & Symbols & Values \\
\hline Input Voltage & $E$ & $40 \mathrm{~V}$ \\
Reference Angular Velocity & $\omega^{*}$ & $150 \mathrm{rad} / \mathrm{s}$ \\
Converter Inductance & $L_{0}$ & $10 \mathrm{mH}$ \\
Converter Capacitance & $C_{0}$ & $1000 \mu \mathrm{F}$ \\
Load Resistance & $R_{0}$ & $250 \Omega$ \\
Armature Inductance & $L_{a}$ & $2 \mathrm{mH}$ \\
Armature Resistance & $R_{a}$ & $1.45 \Omega$ \\
Torque constant & $k_{m}$ & 0.0699 \\
Counter electromotive force constant & $k_{e}$ & 0.0699 \\
Moment of Inertia of Rotor & $J_{0}$ & $32.5 \times 10^{-6}$ \\
Viscous Friction Coefficient & $b$ & $65.12 \times 10^{-6}$ \\
\hline
\end{tabular}

as well as other two popular MPC approaches, namely, ESOenhanced MPC approach (MPC+ESO), and incremental MPC based on a state observer (IMPC+OB) [37]. For simplicity, the proposed approach is called MPC+GPIO subsequently. The $\mathrm{MPC}+\mathrm{ESO}$ approach is employed to verify the advantages of the proposed discrete-time reduced-order GPIO as compared with the traditional ESO, whereas the IMPC+OB approach is implemented to demonstrate the promising performance of the proposed MPC+GPIO against the well-known integral MPC approaches. The basic form of MPC+ESO controller is similar to the method presented in this paper. The only difference lies in that the ESO rather than the GPIO is used to estimate the states and lumped disturbance of the system.

The controller parameters of the proposed MPC+GPIO are assigned as $N_{P}=200$ and $N_{C}=3$. The matrix coefficients
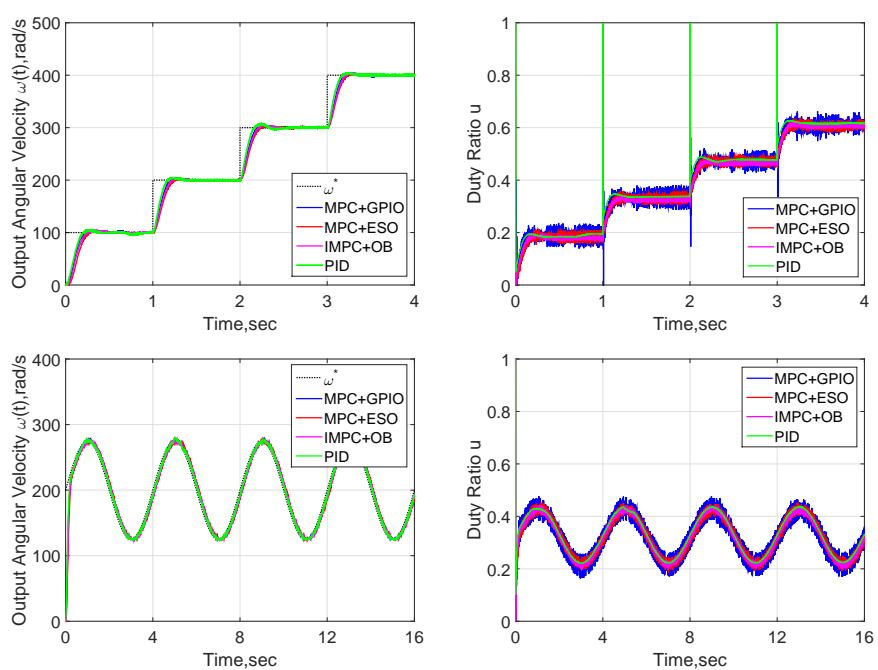

Fig. 4. Nominal speed regulation profile of the four controllers (top: multiple step responses; bottom: time-varying tracking responses; left: angular velocity; right: duty ratio).

of GPIO are designed as

$$
F=\left[\begin{array}{ccccc}
-0.2 & 0.0003 & 0 & 0 & 0 \\
-1920 & 1 & 0.0003 & 0 & 0 \\
-1.536 \times 10^{6} & 0 & 1 & 0.0003 & 0 \\
-6.144 \times 10^{8} & 0 & 0 & 1 & 0.0003 \\
-9.8304 \times 10^{10} & 0 & 0 & 0 & 1
\end{array}\right],
$$

and $G=\left[-2.88 \times 10^{3},-6.144 \times 10^{6},-5.529 \times\right.$ $\left.10^{9},-2.3593 \times 10^{1} 2,-3.9322 \times 10^{14}\right]^{T}$, $H=\left[0,0,1.2905 \times 10^{9}, 0,0\right]^{T}$, and $N=$ $\left[4000,6.4 \times 10^{6}, 5.12 \times 10^{9}, 2.04 \times 10^{1} 2,3.2768 \times 10^{14}\right]^{T}$.

The controller parameters of MPC+ESO are designed as $N_{P}=100$ and $N_{C}=2$. The gains of ESO are selected as $\lambda_{1}=3300, \lambda_{2}=4.34 \times 10^{6}, \lambda_{3}=2.84 \times 10^{9}, \lambda_{4}=$ $9.261 \times 10^{12}, \quad \lambda_{5}=1.2 \times 10^{14}$. The parameters of the IMPC+OB algorithm are designed as $N_{P}=30, N_{C}=1$, $\lambda_{1}=429.2, \quad \lambda_{2}=1.807 \times 10^{3}, \lambda_{3}=1.366 \times 10^{3}, \quad \lambda_{4}=$ $3.269 \times 10^{3}$. The PID controller parameters are assigned as $k_{p}=0.0072, k_{i}=0.06$ and $k_{d}=1 \times 10^{-5}$.

As is well known, there are usually a number of conflicts/constraints in control system design and tuning (e.g., tracking versus disturbance rejection and nominal performance versus robustness). As such, it is generally impossible to design and tune a controller with all performances better than others. For fair comparisons, one reasonable manner is to tune the controllers that obtains some similar performance specifications and then compare the others. Since this paper is mainly concerned with multiple sources of disturbances/uncertainties attenuation, for fair performance comparisons of the four controllers, the control parameters of each controller are tuned such that the speed tracking profiles have similar performance specifications, i.e., settling times and overshoots, in the absence of disturbances and uncertainties. The robustness and disturbance rejection performances are then fairly compared. The nominal speed regulation and tracking profiles under the four controllers are shown in Fig. 4. In the top of the 
figure, the multiple step speed regulation is tested, whereas the time-varying speed tracking profiles are shown in the bottom of the figure where the reference speed is taken as $\omega^{*}(t)=200+75 \sin (0.5 \pi t) \mathrm{rad} / \mathrm{s}$. It can be observed from Fig. 4 that the four controllers have obtained very similar speed regulation and tracking performances in nominal case. The duty ratio profiles of the four controllers also have similar shapes as shown by Fig. 4. This indicates that all the four controllers are well-tuned to behave satisfactory nominal speed regulation performances for fair comparisons.

In what follows, we consider the robustness tests of the four controllers in the presence of various multiple disturbances and uncertainties.

\section{A. Case I-Robustness To Sudden Input Voltage Changes}

In this case, we suppose that the input voltage has a sudden decrease and increase along the following setting

$$
E= \begin{cases}40 \mathrm{~V} \text { (Nominal), } & \text { for } t \in[0,1) \mathrm{sec}, \\ 30 \mathrm{~V} \text { (Decrease), } & \text { for } t \in[1,3) \mathrm{sec}, \\ 40 \mathrm{~V} \text { (Increase), } & \text { for } t \in[3,4] \mathrm{sec}\end{cases}
$$

The response curves of the angular velocity, the armature current, and the profile of the duty ratio under the four controllers are shown in Fig. 5 for this case. To evaluate the performance specification qualitatively, the performance indices including maximum angular velocity drop/raise (MAVD/MAVR) and recovery time (RT) are utilized for performance validation. The corresponding speed regulation performance indices are shown in Table II.

As shown by Fig. 5, all the four controllers could remove the offset caused by the input voltage mutations. However, it can be observed from Fig. 5 and Table II that the PID controller results in large velocity raise and drop as well as long settling time when input voltage changes suddenly. The other three controllers have significantly improved the performance indices of MAVD, MAVR and RT, while the proposed method has exhibited the best speed regulation performance.

\section{B. Case II-Robustness Against Load Torque Variations}

The robustness against load torque variations is tested for the four controllers in this subsection. We assume that the load torque has the following form

$$
\tau_{L}= \begin{cases}0 \text { N.m (Nominal), } & \text { for } t \in[0,1) \mathrm{sec}, \\ 0.1 \text { N.m (Load On), } & \text { for } t \in[1,3) \mathrm{sec}, \\ 0 \text { N.m (Load Off), } & \text { for } t \in[3,5] \mathrm{sec} .\end{cases}
$$

The profiles of the angular velocity, the armature current and the duty ratio of the experimental tests are plotted in Fig. 6. The corresponding performance indices under the four controllers in Case II are given in Table II.

Again it can be observed from Fig. 6 and Table II that the proposed MPC+GPIO has attained the best performance among the four controllers.
TABLE II

Performance Indices in Three Uncertain Cases

\begin{tabular}{c|c|c|c|c}
\hline \multirow{2}{*}{ Test Type } & \multirow{2}{*}{ Controllers } & \multicolumn{3}{|c}{ Performance } \\
\cline { 3 - 5 } & & MAVD (rad/s) & MAVR (rad/s) & RT (sec) \\
\hline \multirow{3}{*}{ CASE I } & MPC+GPIO & 28.7 & 35.7 & 0.419 \\
\cline { 2 - 5 } & MPC+ESO & 33 & 44.1 & 0.448 \\
\cline { 2 - 5 } & IMPC+OB & 31.9 & 39.1 & 0.416 \\
\cline { 2 - 5 } & PID & 47 & 77 & 0.697 \\
\hline \multirow{3}{*}{ CASE II } & MPC+GPIO & 7.1 & 7.9 & 0.917 \\
\cline { 2 - 5 } & MPC+ESO & 8.9 & 12.8 & 0.977 \\
\cline { 2 - 5 } & IMPC+OB & 11.5 & 16.1 & 0.899 \\
\cline { 2 - 5 } & PID & 19.1 & 20.5 & 1.022 \\
\hline \multirow{3}{*}{ CASE III } & MPC+GPIO & 2.1 & 4.8 & - \\
\cline { 2 - 5 } & MPC+ESO & 5.5 & 8.3 & - \\
\cline { 2 - 5 } & IMPC+OB & 6.1 & 10 & - \\
\cline { 2 - 5 } & PID & 9.1 & 14.9 & - \\
\hline
\end{tabular}

\section{Case III-Robustness Against Time-Varying Distur- bances}

We further investigate the robustness against time-varying disturbances of the four controllers in this case. Specifically, a generic sawtooth waveform of time-varying load torque disturbance is taken to acting on the DC motor. The frequency and amplitude of the load torque are $1 \mathrm{~Hz}$ and 0.15 N.m, respectively. Response curves of the angular velocity, the armature current and the duty ratio in the presence of such a time-varying disturbance under the four controllers are shown in Fig. 7. The performance indices are shown in Table II.

As shown by Fig. 7 and Table II, one can observe that the PID controller has led to large steady-state speed perturbations. The MPC+ESO and IMPC+OB have improved a lot on robustness performance against time-varying load torque but still caused considerable speed fluctuations. The proposed MPC+GPIO has significantly reduced the speed fluctuation as compared to other two MPC algorithms. It can be observed from Table II that the MAVD and MAVR under the proposed $\mathrm{MPC}+\mathrm{GPIO}$ approach are only 2.1 and $4.8 \mathrm{rad} / \mathrm{s}$ respectively, which are about half of these of $\mathrm{MPC}+\mathrm{ESO}$ and IMPC+OB approaches, and one third of these of the PID controller.

\section{CONCLUSION}

This paper has proposed an almost senseless discrete-time robust predictive speed regulation algorithm for a generic DCDC buck power converter-driven DC motor. A discrete-time reduced-order GPIO has been proposed to estimate unmeasurable virtual states and lumped disturbances and uncertainties. With the help of GPIO, the speed in the future prediction horizon has been predicted to facilitate MPC design. The input constraint on duty ratio has been imposed on the receding horizon optimization process, which finally gives the robust predictive speed regulation law. The experimental results on the DSPACE real-time control test setup have shown that the proposed MPC+GPIO method outperforms PID controller and other two MPC approaches in the presence of various disturbances and uncertainties. A promising future research direction is to extend the proposed method to bidirectional converter-driven DC motor system. 

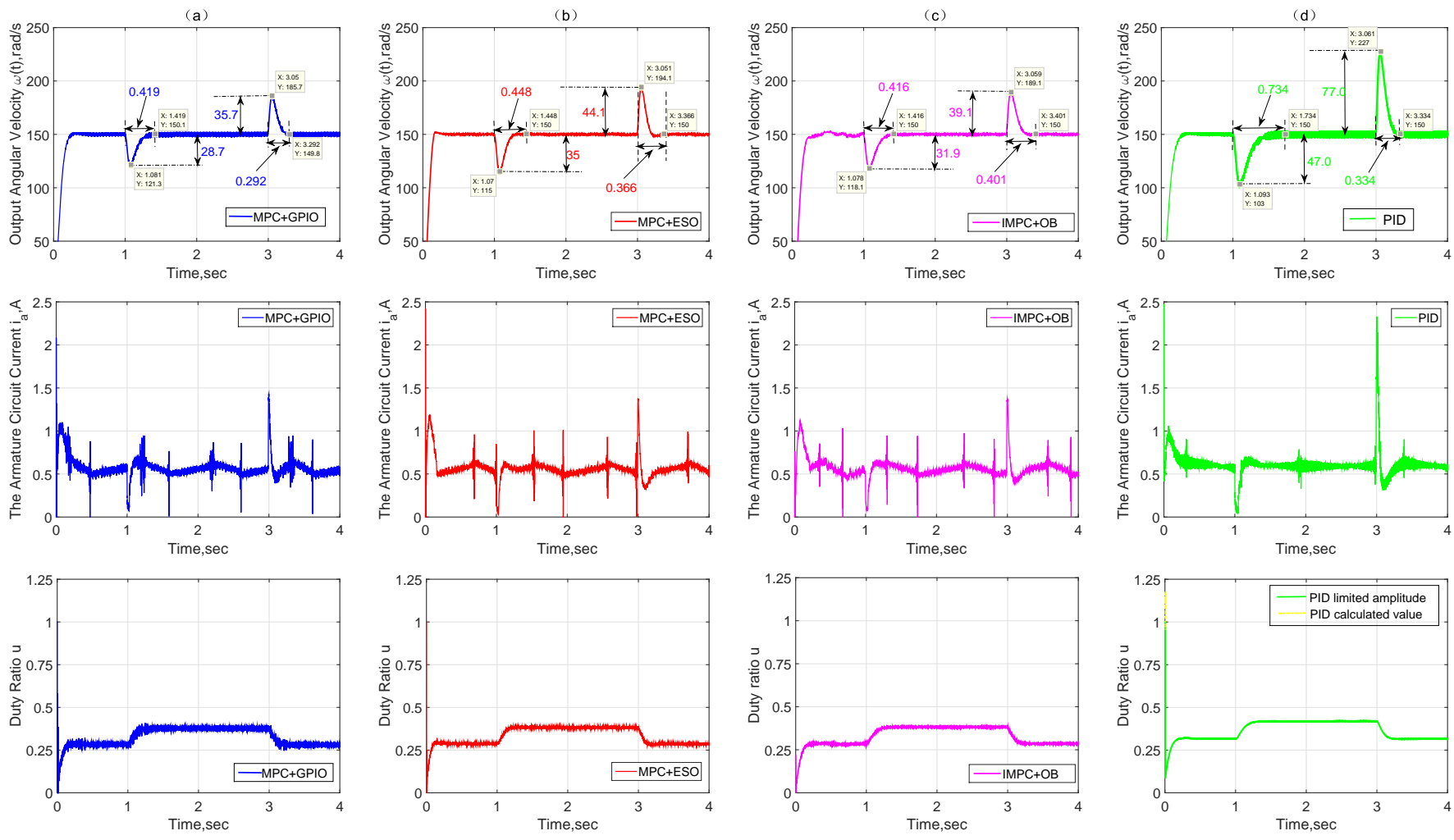

Fig. 5. Case I: Variable response curves of the converter-driven DC motor under MPC+GPIO (a), MPC+ESO (b), IMPC+OB (c) and PID (d) approaches in the presence of sudden input voltage changes (top: angular velocity; middle: armature current; bottom: duty ratio).

\section{REFERENCES}

[1] I. Boldea and S. A. Nasar, Electric Drives (second edition). CRC Press, 2005.

[2] S. E. Lyshevski, Electromechanical Systems, Electric Machines, and Applied Mechatronics. CRC Press, 2000.

[3] P. Krause, O. Wasynczuk and S. Sudhoff, Analysis of Electric Machinery and Drive Systems. Wiley-IEEE Press, 2013.

[4] P. Pillay and R. Krishnan, "Modeling, simulation, and analysis of permanent-magnet motor drives. II. The brushless DC motor drive," IEEE Transactions on Industry Applications, vol. 25, no. 2, pp. 274-279, 1989.

[5] R. S. Ortigoza, V. M. H. Guzman, M. A. Cruz and D. M. Carrillo, "DC/DC buck power converter as a smooth starter for a dc motor based on a hierarchical control," IEEE Transactions on Power Electronics, vol. 30, no. 2, pp. 1076-1084, 2014.

[6] H. S. Ramirez and M. A. O. Salazar, "On the robust control of buck-converter DC-motor combinations," IEEE Transactions on Power Electronics, vol. 28, no. 8, pp. 3912-3922, 2013.

[7] J. L. Flores, J. Reger and H. S. Ramirez, "Load torque estimation and passivity-based control of a boost-converter/DC-motor combination," IEEE Transactions on Control Systems Technology, vol. 18, no. 6, pp. $1398-1405,2010$

[8] J. L. Flores, J. L. B. Avalos, H. S. Ramirez and M. A. C. Ordaz, "Robust passivity-based control of a buck-boost converter/DC-motor system: An active disturbance rejection approach," IEEE Transactions on Industry Applications, vol. 48, no. 6, pp. 2362-2371, 2013.

[9] S. G. Kumar and S. H. Thilagar, "Sensorless load torque estimation and passivity based control of buck converter fed DC motor," The Scientific World Journal, vol. 2015, pp. 1-15, 2015.

[10] M. A. Ahmad, R. M. T. Raja-Ismail and M. S. Ramli, "Control strategy of buck converter driven DC motor: A comparative assessment," Australian Journal of Basic and Applied Sciences, vol. 4, no. 10, pp. 4893-4903, 2010

[11] R. S. Ortigoza, J. R. Garcia, J. M. A. Martinez, V. M. H. Guzman, M. M. Aranda, H. Taud and R. B. Quintero, "Two-stage control design of a buck converter/DC motor system without velocity measurements via a $\Sigma$ - $\Delta$-modulator," Mathematical Problems in Engineering, vol. 2013, pp. 1-11, 2013.

[12] R. S. Ortigoza, C. M. Sanchez, F. C. Corral, M. A. Cruz, J. M. A Martinez and G. S. Gonzalez, "Hierarchical velocity control based on differential flatness for a DC/DC Buck converter-DC motor system," Mathematical Problems in Engineering, vol. 2014, pp. 1-12, 2014.

[13] V. M. H. Guzman, R. S. Ortigoza and D. M. Carrillo, "Velocity control of a brushed DC-motor driven by a DC to DC buck power converter," International Journal of Innovative Computing, Information and Control, vol. 11, no. 2, pp. 509-521, 2015.

[14] S. Khubalkar, A. Chopade, A. Junghare, M. Aware and S. Das, "Design and realization of stand-alone digital fractional order PID controller for Buck converter fed DC motor," Circuits, Systems, and Signal Processing, vol. 35 , no. 6 , pp. $2189-2211,2016$.

[15] G. Rigatos, P. Siano, P. Wira and M. Sayed-Mouchaweh, "Control of DC-DC converter and DC motor dynamics using differential flatness theory," Intelligent Industrial Systems, vol. 2, no. 4, pp. 371-380, 2016.

[16] T. K. Nizami, A. Chakravarty and C. Mahanta, "Design and implementation of a neuro-adaptive backstepping controller for Buck converter fed PMDC-motor," Control Engineering Practice, vol. 58, pp. 78-87, 2017.

[17] S. Malek, "A new nonlinear controller for DC-DC boost converter fed DC motor," International Journal of Power Electronics, vol. 7, no. 1-2, pp. 54-71, 2015.

[18] G. C. Konstantopoulos and A. T. Alexandridis, "Enhanced control design of simple DC-DC boost converter-driven DC motors: Analysis and implementation," Electric Power Components and Systems, vol. 43, no. 17, pp. 1946-1957, 2015.

[19] A. T. Alexandridis and G. C. Konstantopoulos, "Modified PI speed controllers for series-excited DC motors fed by DC/DC boost converters," Control Engineering Practice, vol. 23, pp. 14-21, 2014.

[20] R. S. Ortigoza, J. N. A. Juarez, J. R. G. Sanchez, M. A. Cruz, V. M. H. Guzman and H. Taud, "Modeling and experimental validation of a bidirectional DC/DC Buck power electronic converter-DC motor system," IEEE Latin America Transactions, vol. 15, no. 6, pp. 1043$1051,2017$.

[21] R. S. Ortigoza, J. N. A. Juarez, J. R. G. Sanchez, V. M. H. Guzman, C. Y. S. Cervantes and H. Taud, "A sensorless passivity-based control 

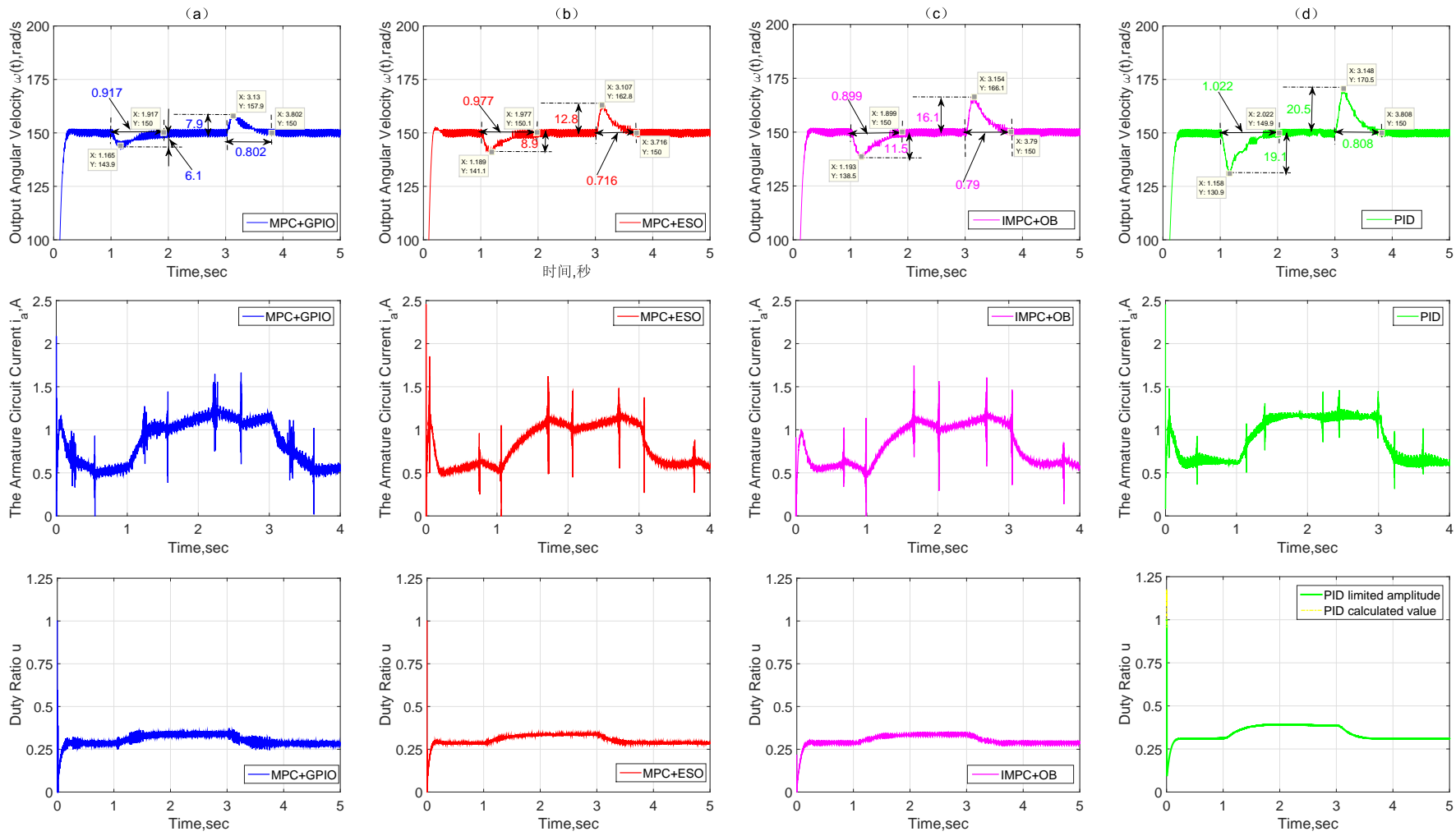

Fig. 6. Case II: Variable response curves of the converter-driven DC motor under MPC+GPIO (a), MPC+ESO (b), IMPC+OB (c) and PID (d) approaches, in the presence of sudden load torque changes (top: angular velocity; middle: armature current; bottom: duty ratio).

for the DC/DC buck converter-inverter-DC motor system," IEEE Latin America Transactions, vol. 14, no. 10, pp. 4227-4234, 2016.

[22] V. H. G. Rodriguez, R. S. Ortigoza, E. H. Marquez, J. R. G. Sanchez, M. P. Silva and G. S. Gonzalez, "A DC motor driven by a DC/DC boost converter-inverter: Modeling and simulation," in Proc. 2016 International Conference on Mechatronics, Electronics and Automotive Engineering, Cuernavaca, Morelos, Mexico, Nov. 22-25, 2016, pp. 78-83.

[23] R. S. Ortigoza, V. H. G. Rodriguez, E. H. Marquez, M. Ponce, J. R. G. Sanchez, J. N. A. Juarez, G. S. Ortigoza and J. H. Perez, "A trajectory tracking control for a boost converter-inverter- DC motor combination," IEEE Latin America Transactions, vol. 16, no. 4, pp. 1008-1014, 2018.

[24] E. H. Marquez, R. S. Ortigoza, J. R. G. Sanchez, V. H. G. Rodriguez and J. N. A. Juarez, "A new DC/DC buck-boost converter-DC motor system: Modeling and experimental validation," IEEE Latin America Transactions, vol. 15, no. 11, pp. 2043-2049, 2017.

[25] E. H. Marquez, R. S. Ortigoza, C. A. A. Rea, J. R. G. Sanchez, M. A Cruz, H. Taud, S. Dong and M. M. Aranda, "Regulation of the DC/DC buck-boost converter-inverter-DC motor system: Sensorless passivity based control," in Proc. 2016 International Conference on Mechatronics, Electronics and Automotive Engineering, Cuernavaca, Morelos, Mexico, Nov. 21-24, 2017, pp. 88-92.

[26] J. L. Flores, H. S. Ramirez, E. F. C. Lopez and M. A. C. Ordaz, "Sensorless passivity based control of a DC motor via a solar powered Sepic converter-full bridge combination," Journal of Power Electronics, vol. 11, no. 5, pp. 743-750, 2011.

[27] H. Wu, L. Zhang, J. Yang and S. Li, "Model predictive control for DCDC buck power converter-DC motor system with uncertainties using a GPI observer," the 36th Chinese Control Conference, pp. 4906-4911, 2017.

[28] F. Wei, P. Yang and W. Li, "Robust adaptive control of DC motor system fed by buck converter,' International Journal of Control and Automation, vol. 7, no. 10, pp. 179-190, 2014.

[29] G. Gateau, P. Maussion and J. Faucher, "Fuzzy controller based on optimal control commutation principle for a DC/DC converter in current mode," in International Conference on Industrial Electronics, Control and Instrumentation, pp. 1320-1324, vol.2, 1994.

[30] D. Grignion, X. Chen, N. Kar and H. Qian, "Estimation of load disturbance torque for DC motor drive systems under robustness and sensitivity consideration," IEEE Transactions on Industrial Electronics, vol. 61, no. 2, pp. 930-942, 2013.

[31] K. R. Muske and J. B. Rawlings, "Model predictive control with linear models," AIChE Journal, vol. 39, no. 2, pp. 262-287, 1993.

[32] H. Liu and S. Li, "Speed control for PMSM servo system using predictive functional control and extended state observer," IEEE Transactions on Industrial Electronics, vol. 59, no. 2, pp. 1171-1183, 2011.

[33] J. D. Barros, J. F. A. Silva and E. G. A. Jesus, "Fast-predictive optimal control of NPC multilevel converters," IEEE Transactions on Industrial Electronics, vol. 60, no. 2, pp. 619-627, 2013.

[34] C. Xia, M. Wang, Z. Song and T. Liu, "Robust model predictive current control of three-phase voltage source PWM rectifier with online disturbance observation," IEEE Transactions on Industrial Informatics, vol. 8, no. 3, pp. 459-471, 2012.

[35] C. Westermayer, R. Priesner, M. Kozek and R. Bauer, "High dynamic torque control for industrial engine test beds," IEEE Transactions on Industrial Electronics, vol. 60, no. 9, pp. 3877-3888, 2013.

[36] J. Yang, W. X. Zheng, S. Li, B. Wu and M. Cheng, "Design of a prediction-accuracy-enhanced continuous-time MPC for disturbed systems via a disturbance observer," IEEE Transactions on Industrial Electronics, vol. 62, no. 9, pp. 5807-5816, 2015.

[37] L. Wang, S. Chai, D. Yoo, L. Gan and K. Ng, PID and Predictive Control of Electrical Drives and Power Converters Using MATLAB/Simulink. Wiley-IEEE Press, 2015.

[38] Z. Ding, "Asymptotic rejection of unknown sinusoidal disturbances in nonlinear systems," Automatica, vol. 43, no. 1, pp. 174-177, 2007.

[39] J. Su and W.-H. Chen, "Further results on 'Reduced order disturbance observer for discrete-time linear systems'," Automatica, vol. 93, pp. 550553,2018

[40] L. Wang, S Chai, D. Yoo, L. Gan and K. Ng, Discrete-time Model Predictive Control (DMPC) of Electrical Drives and Power Converter. Wiley-IEEE Press, 2015.

[41] M. Fliess, R. Marquez, E. Delaleau and H. S. Ramirez, "Generalized proportional-integral controllers," ESAIM: Control, Optimisation and Calculus of Variations, vol. 7, pp. 23-41, 2002.

[42] J. Han, "From PID to active disturbance rejection control," IEEE Transactions on Industrial Electronics, vol. 56, no. 3, pp. 900-906, 2009. 

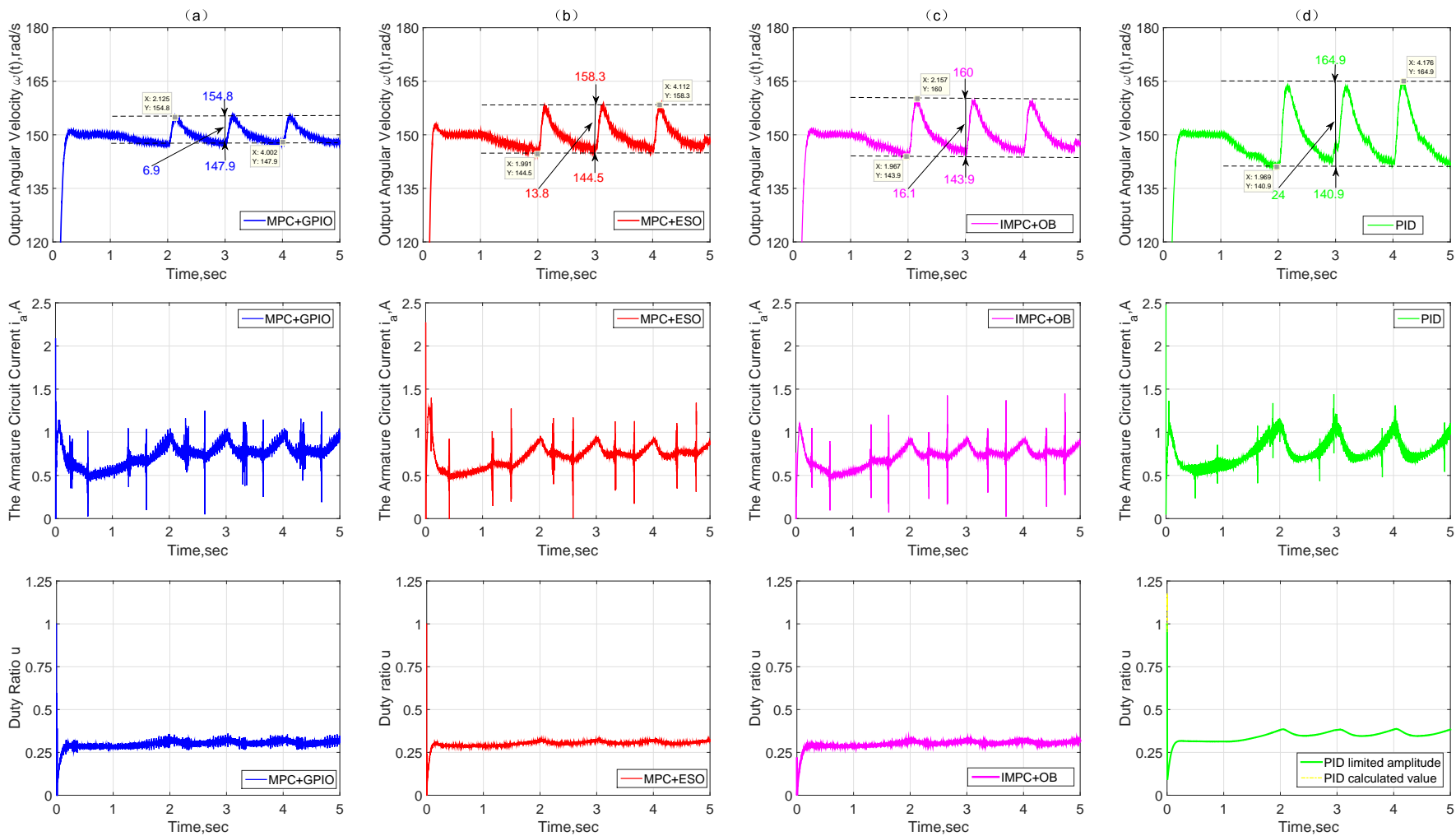

Fig. 7. Case III: Variable response curves of the converter-driven DC motor under MPC+GPIO (a), MPC+ESO (b), IMPC+OB (c) and PID (d) approaches, in the presence of time-varying load torque changes (top: angular velocity; middle: armature current; bottom: duty ratio).

[43] B. Xu, D. Wang, Y. Zhang and Z. Shi, "DOB-based neural control of flexible hypersonic flight vehicle considering wind effects," IEEE Transactions on Industrial Electronics, vol. 64, no. 11, pp. 8676-8685, 2017.

[44] X.J. Wei, Z.J. Wu, and H.R. Karimi, "Disturbance observer-based disturbance attenuation control for a class of stochastic systems," Automatica, vol. 63, pp. 21-25, 2016

[45] L. Wang. Model Predictive Control System Design and Implementation Using MATLAB. Springer Science \& Business Media, 2009.

[46] B. W. Bequette. Process Control: Modeling, Design and Simulation. Prentice Hall, 2003. 